\title{
Efectos de la temperatura sobre el crecimiento individual y formación de zooides en Stylaria lacustris (Linnaeus, 1767) (Oligochaeta, Naididae)
}

\author{
Laura C. Armendáriz
}

Biota Neotropica $v 7$ (n2) - http://www.biotaneotropica.org.br/v7n2/pt/abstract?article +bn00907022007

\author{
Recebido em 16/10/06 \\ Versão reformulada recebida em $27 / 02 / 07$ \\ Publicado em 01/05/07 \\ División Zoología Invertebrados, Facultad de Ciencias Naturales y Museo, \\ UNLP. Paseo del Bosque S/N, La Plata, 1900, Argentina \\ e-mail: lauraarm@netverk.com.ar
}

\begin{abstract}
Armendáriz, L.C. Temperature effects on individual growth and zooids formation in Stylaria lacustris (Linnaeus, 1767) (Oligochaeta, Naididae) Biota Neotrop. May/Aug 2007 vol. 7, no. 2 http://www.biotaneotropica. org.br/v7n2/pt/abstract?article+bn00907022007. ISSN 1676-0603.

The experiments carried out with $S$. lacustris showed that the temperature plays a role of importance in the rate of asexual reproduction, as for the number of zooids produced by individual, as well as to the size reached by them. On the other hand, it would regulate the rate of growth and the sexual reproduction, giving place to the maturity of the individuals when temperature increases. The life time of the immature individuals was estimated in 15 days, being this independent of the temperature used in the cultures. The mature individuals lived on the average 37 days. The survival in S. lacustris was falling gradually, following a curve of type 1ll. Life expectancy presented its major value at the age of 16 days and stayed approximately constant up to an age of 28 days, after then falled persistently until the last reached age. The individual growth according to von Bertalanffy equation, was estimated for the organisms maintained at $23 \pm 2{ }^{\circ} \mathrm{C}$ and at $14 \pm 1{ }^{\circ} \mathrm{C}$. Those individuals cultivated at higher temperature attained a bigger size, although their rate of growth was smaller than those maintained at $14{ }^{\circ} \mathrm{C}$.

Keywords: cultures, asexual reproduction, Argentina.
\end{abstract}

\section{Resumen}

Armendáriz, L.C. Efectos de la temperatura sobre el crecimiento individual y formación de zooides en Stylaria lacustris (Linnaeus, 1767) (Oligochaeta, Naididae) Biota Neotrop. May/Aug 2007 vol. 7, no. 2 http:// www.biotaneotropica.org.br/v7n2/pt/abstract?article+bn00907022007. ISSN 1676-0603.

Los cultivos realizados con S. lacustris demostraron que la temperatura juega un papel de importancia en la tasa de la reproducción, en cuanto al número de zooides producido por individuo así como también al tamaño alcanzado por los mismos. Por otro lado, regularía la tasa de crecimiento y la reproducción sexual, dando lugar a la madurez de los individuos cuando aumenta la temperatura. El tiempo de vida promedio de los individuos inmaduros se estimó en 15 días, siendo este valor independiente de la temperatura utilizada en los cultivos. Los individuos maduros vivieron en promedio 37 días. La supervivencia en S. lacustris fue decreciendo gradualmente, según una curva de tipo 11l. La expectativa de vida presentó su pico a la edad de 16 días y se mantuvo aproximadamente constante hasta una edad de 28 días a partir de la cual fue decreciendo en forma persistente hasta la última edad alcanzada. Se estimó el crecimiento individual, según la ecuación de von Bertalanffy, para los organismos mantenidos a $23 \pm 2{ }^{\circ} \mathrm{C}$ y a $14 \pm 1{ }^{\circ} \mathrm{C}$. En aquellos individuos cultivados a mayor temperatura se registró una mayor talla, aunque su tasa de crecimiento fue menor que la de los individuos mantenidos a $14{ }^{\circ} \mathrm{C}$.

Palabras-clave: cultivos, reproducción asexual, Argentina. 


\section{Introducción}

Los oligoquetos acuáticos de la familia Naididae presentan una amplia distribución mundial con adaptaciones a un extenso rango de condiciones ambientales. La mayoría habita ambientes acuáticos continentales, con especies adaptadas incluso a aguas salobres y suelos húmedos o pantanosos. En América del Sur son un componente común en las diferentes comunidades acuáticas continentales asociados a distintos sustratos: macrofitas, algas filamentosas, sedimentos, rocas, como así también en sustratos artificiales (Brinkhurst \& Jamieson 1971, Brinkhurst \& Marchese 1992). Debido a su sensibilidad a diversos agentes contaminantes han sido utilizados como indicadores de eutrofización, así como también en la construcción de índices para evaluar polución y calidad de aguas (Särkkä 1987, Sang 1987, Lang 1990, Milbrink 1994).

Los naidídeos son consumidos por los organismos depredadores de la comunidad, constituyendo un eslabón importante en la transferencia de energía a los niveles tróficos superiores. Son empleados como fuente de alimento en acuicultura comercial, pero también representan una importante vía en la transferencia de tóxicos de los niveles tróficos inferiores a los superiores (Lietz 1987).

En cuanto a su biología son organismos oportunistas que presentan dos estrategias reproductivas: asexual por fisión y sexual, revelando una alta plasticidad biológica. La primera estrategia ocurre en condiciones ambientales favorables y provoca un rápido crecimiento poblacional. La segunda, en cambio, es utilizada comunmente en condiciones desfavorables (Christensen 1984, Juget \& Lafont 1994, Armendáriz 1999, 2000). La reproducción asexual puede realizarse por medio de dos mecanismos, paratomía y arquitomía. En el primer caso, se forma una zona de brotación a partir de la cual se originará un nuevo individuo, que formará su extremo anterior previamente a su separación del individuo progenitor. Es quizás el modo de reproducción más frecuente y los individuos forman cadenas de zooides en número variable. Estas cadenas se manifiestan externamente a partir de constricciones más o menos profundas en la región media del cuerpo, donde el zooide en formación llega a mostrar cierto grado de cefalización, previamente a su separación del individuo progenitor (Brinkhurst \& Jamieson 1971, Learner et al. 1978, Christensen 1984).

En un caso típico, la alternancia de reproducciones asexual y sexual sigue en naidídeos un ciclo anual. Las poblaciones presentan grandes fluctuaciones estacionales, creciendo mediante explosiones regulares o erráticas que alcanzan altas densidades cuando las condiciones ambientales son favorables debido, en parte, a la reproducción asexual anteriormente mencionada (Loden 1981, Irmler 1989, Becket et al. 1992, Sloreid 1994). Los factores que influyen en la tasa de reproducción asexual son la conductividad, el tenor de oxígeno del agua, la duración del fotoperíodo, la disponibilidad de alimento y principalmente la temperatura (Loden 1981, Smith 1986, Schierwater \& Hauenschild 1990a, b, Lochhead \& Learner 1983).

La generación sexual aparece en general, en ambientes naturales, antes del invierno o período de sequía y culmina en la deposición de las ootecas o cocones. A menudo los individuos mueren luego de la puesta que, en el caso particular de Stylaria lacustris (Linnaeus, 1767), correspondería a una edad de 1-2 meses. En algunas especies esta reproducción es muy rara y en cierta forma errática en su ocurrencia, con frecuencia es de breve duración y la maduración de los individuos es sincrónica (Brinkhurst \& Jamieson 1971, Poddubnaya 1972, Christensen 1984, Timm 1984, Smith 1986, Armendáriz 1999, 2000).

S. lacustris es una especie cosmopolita, tolerante a fluctuaciones ambientales y con amplitud trófica (Learner et al. 1978, Särkkä 1987, Sloreid 1994). En Argentina su dinámica poblacional ha sido estudiada en cuerpos de agua vegetados cercanos al Río de la Plata (Armendáriz 2000). El objetivo de este trabajo fue llevar a cabo experiencias de laboratorio a fin de determinar sus tiempos generacionales y producción de zooides, la supervivencia, expectativa de vida y complementar los datos obtenidos en campo para el análisis de crecimiento.

\section{Materiales y Métodos}

Stylaria lacustris fue colectada en una charca vegetada de la localidad de Los Talas, partido de Berisso, a los $34^{\circ} 53^{\prime} \mathrm{S}$ y $57^{\circ} 50^{\prime} \mathrm{W}$, y próxima al Río de La Plata. La carpeta de vegetación flotante estuvo constituida por Spirodela intermedia (Koch.).

Los ejemplares fueron transportados in vivo al laboratorio, donde se realizaron cultivos durante cuatro meses. Se utilizaron cajas de Petri con una delgada capa de arena y $1,5-2 \mathrm{~cm}$ de agua mineralizada. En cada recipiente se mantuvieron uno a cuatro individuos, que fueron alimentados con lechuga macerada y alimento para peces. Los cultivos se realizaron bajo dos condiciones experimentales: en un caso (E1) se mantuvieron durante un mes a temperatura ambiente $\left(23 \pm 2{ }^{\circ} \mathrm{C}\right)$ y en un segundo caso (E2) se mantuvieron durante tres meses a una temperatura de $14 \pm 1{ }^{\circ} \mathrm{C}$ (Learner et al. 1978, Streit 1978, Finogenova 1984, Schierwater \& Hauenschild 1990a, Juget \& Lafont 1994). Los organismos fueron medidos diariamente bajo microscopio estereoscópico, para ello se colocaron en vidrios de reloj con agua helada a fin de enlentecer sus movimientos, registrándose los incrementos en longitud así como también los nacimientos y muertes. Cada dos días se cambió además el agua junto con el alimento. Las diferencias entre ambas experiencias fueron analizadas utilizando el test de Student, previa evaluación de homogeneidad de varianzas mediante la prueba de F (Snedecor \& Cochran 1984). Se construyó una tabla de vida a fin de determinar en los cultivos la supervivencia y expectativa de vida.

Se calcularon curvas teóricas de crecimiento para los individuos mantenidos bajo las dos condiciones de laboratorio antes citadas, según la ecuación de von Bertalanffy (Lévêque 1971):

$$
\mathrm{Lt}=\mathrm{L} \propto\left(1-\mathrm{e}^{-\mathrm{k}(\mathrm{t}-\mathrm{to})}\right)
$$

donde Lt es la talla del individuo al tiempo t, L $\propto$ el valor de Lt cuando la tasa de crecimiento es nula, $\mathrm{k}$ la tasa de crecimiento, $\mathrm{t}$ la edad (expresada en días) y to es el tiempo hipotético en que el individuo tiene una talla igual a 0 .

\section{Resultados}

A partir de siete ejemplares colectados (seis inmaduros y uno maduro) se obtuvieron, al cabo de cuatro meses de cultivos, 136 individuos.

En la Tabla 1 se indican la longevidad media (estimada en días), el número promedio de zooides producidos por individuo, el tiempo empleado en tener lugar la reproducción asexual (desde el inicio de la zona de brotación hasta la separación de los dos individuos), así como también la longitud máxima alcanzada por la cadena de zooides. En la primera experiencia (E1) la cadena de zooides alcanzó, al momento de la división, una talla promedio de $11,40 \mathrm{~mm}$, estimandose la talla del individuo progenitor en 7,53 $\mathrm{mm}$ y la del nuevo individuo en 3,86 mm. Si bien durante esta experiencia 12 individuos maduraron sexualmente, no pudo observarse deposición de cocones $\mathrm{u}$ ootecas. En estos individuos maduros la longevidad fue mayor que en los inmaduros ( $\mathrm{x}=36,90$ días; desvío estandar $=32,81$; tiempo mínimo 12 y máximo 111 días). En estos ejemplares, al alcanzar una talla de 10-11 mm, se hizo evidente la formación del clitelo. La 
Tabla 1. Cultivos de Stylaria lacustris mantenidos a $23 \pm 2{ }^{\circ} \mathrm{C} \mathrm{y} 14 \pm 1{ }^{\circ} \mathrm{C}$. En $*$ las diferencias son significativas al valor de $\mathrm{t}$ (p $<0,05$ ).

Table 1. Cultures of Stylaria lacustris maintained at $23 \pm 2{ }^{\circ} \mathrm{C}$ and $14 \pm 1{ }^{\circ} \mathrm{C}$. In $*$ the differences are significant to the value of $\mathrm{t}$ (p $<0,05$ ).

\begin{tabular}{|c|c|c|}
\hline & Cultivos a $23 \pm 2{ }^{\circ} \mathrm{C}$ & Cultivos a $14 \pm 1^{\circ} \mathrm{C}$ \\
\hline Longevidad media (días) & $\begin{array}{l}\mathrm{x}=15,93(\mathrm{n}=29 ; \mathrm{ds}=15,38) \\
\operatorname{mí}=4 \\
\max =90\end{array}$ & $\begin{array}{l}x=15,61(\mathrm{n}=54 ; \mathrm{ds}=16,62) \\
\operatorname{mí}=2 \\
\max =75\end{array}$ \\
\hline * Zooides producidos & $\begin{array}{l}x=1,35(\mathrm{n}=17 ; \mathrm{ds}=0,60) \\
\text { mín }=1 \\
\text { máx }=3\end{array}$ & $\begin{array}{l}x=2,033(\mathrm{n}=30 ; \mathrm{ds}=1,06) \\
\text { mín }=1 \\
\operatorname{máx}=5\end{array}$ \\
\hline * Tiempo empleado en la reproducción asexual (días) & $\begin{array}{l}x=5,6(n=20 ; d s=3,97) \\
\text { mín }=1 \\
\text { máx }=16\end{array}$ & $\begin{array}{l}\mathrm{x}=7,92(\mathrm{n}=71 ; \mathrm{ds}=4,55) \\
\text { mín }=2 \\
\operatorname{máx}=30\end{array}$ \\
\hline * Longitud alcanzada por la cadena de zooides (mm) & $\begin{array}{l}\mathrm{x}=11,4(\mathrm{n}=26 ; \mathrm{ds}=2,40) \\
\text { mín }=8,5 \\
\text { máx }=19\end{array}$ & $\begin{array}{l}\mathrm{x}=13,83(\mathrm{n}=71 ; \mathrm{ds}=2,58) \\
\text { mín }=10 \\
\text { máx }=20\end{array}$ \\
\hline
\end{tabular}

talla máxima registrada para un individuo clitelado fue $20 \mathrm{~mm}$. En algunos organismos clitelados se observó además la presencia de una zona de fisión, si bien dichos individuos murieron antes de concluir la división asexual.

En la segunda experiencia (E2) en cambio, la cadena de zooides alcanzó al momento de su división una talla promedio de $13,83 \mathrm{~mm}$, siendo $9,25 \mathrm{~mm}$ la longitud del individuo progenitor y $4,75 \mathrm{~mm}$ la talla del nuevo individuo. A esta temperatura no se registraron organismos que maduraran sexualmente.

Se aplicó el método de Student para analizar las diferencias entre las dos experiencias realizadas, hallándose diferencias no significativas $(t=-0,085 ; p>0,05)$ en cuanto al tiempo de vida de los individuos, pero sí respecto del número de zooides producidos por individuo $(\mathrm{t}=-2,412, \mathrm{p}<0,05)$, el tiempo estimado empleado en la división $(\mathrm{t}=-2,07, \mathrm{p}<0,05)$ y la longitud alcanzada por la cadena de zooides $(\mathrm{t}=-4,17, \mathrm{p}<0,05)$.

En ambas experiencias, durante la reproducción asexual, los organismos formaron cadenas de dos zooides, rara vez tres. Cuando el zooide en formación alcanzó una longitud de 2-3,5 mm, se hizo evidente la formación de la proboscis, produciéndose luego la separación del nuevo individuo.

A partir de los individuos nacidos durante las dos experiencias, y debido a que la longevidad media no mostró diferencias significativas, fue construída una tabla de vida cuya supervivencia y expectativa de vida se indican en la Figura 1. Se puede observar que la supervivencia decrece gradualmente presentando de este modo una mortalidad proporcional al número de individuos, que correspondería a una curva de tipo 1ll. Respecto a la expectativa de vida, su mayor valor se presenta a los 16 días, edad en que se concentra una alta mortalidad. Se mantiene luego aproximadamente constante hasta la edad de 28 días para posteriormente decrecer en forma persistente hasta la edad última.

En la Figura 2 se indica el crecimiento individual estimado (desde el nacimiento hasta el momento en que comenzó la formación de un zooide por reproducción asexual) para los organismos mantenidos a $23 \pm 2{ }^{\circ} \mathrm{C}$ (tanto los maduros como los inmaduros) y a $14 \pm 1{ }^{\circ} \mathrm{C}$ (inmaduros). En aquellos individuos mantenidos a mayor temperatura se registró una mayor talla, aunque su tasa de crecimiento fue menor que la de los individuos mantenidos a $14{ }^{\circ} \mathrm{C}$.

\section{Discusión}

Distintos factores ambientales afectarían la dinámica poblacional de naidídeos regulando la densidad de individuos,

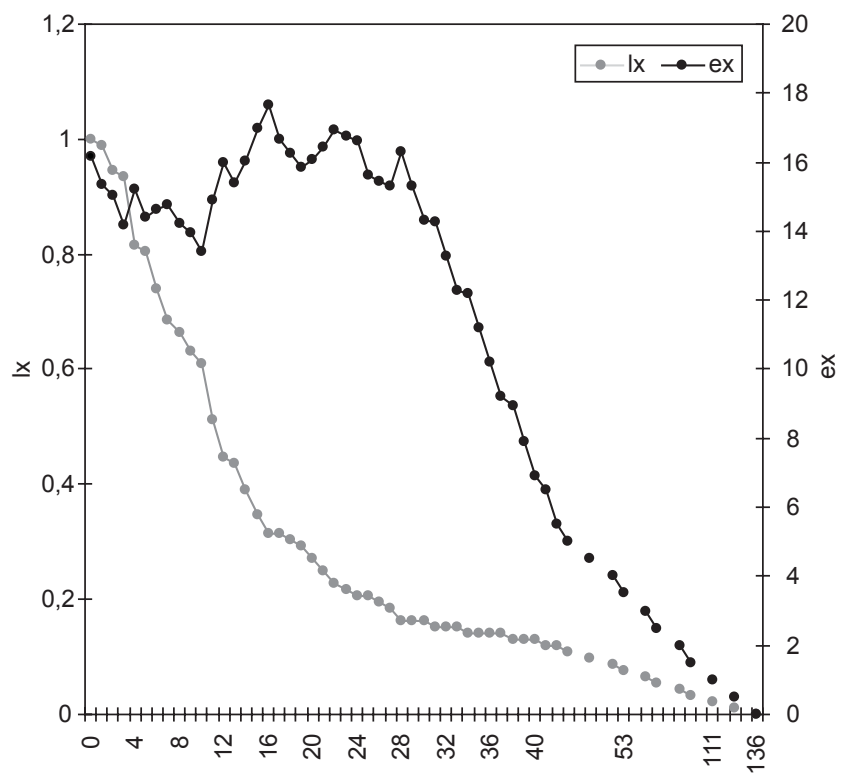

Días

Figura 1. Curvas de supervivencia (lx) y expectativa de vida (ex) en cultivos de Stylaria lacustris.

Figure 1. Survival (1x) and life expectancy (ex) curves in cultures of Stylaria lacustris.

en especial a través de un control en la tasa de reproducción asexual. Entre ellos se destacan: la temperatura, la disponibilidad de alimento, la profundidad, la conductividad, el tenor de oxígeno disuelto y el fotoperíodo (Learner et al. 1978, Loden 1981, Lochhead \& Learner 1983, Smith 1986, Irmler 1989, Schierwater \& Hauenschild 1990a, b). En el caso particular de la temperatura, si bien la mayoría de las especies registran un incremento en su tasa de fisión con el aumento en la misma, en ciertas especies, entre las cuales se encuentra Stylaria lacustris, la mayor abundancia ocurre en ambientes con menor temperatura (Poddubnaya 1972, Learner et al. 1978, Lochhead \& Learner 1983, Smith 1986, Irmler 1989). Tal es el caso de la población de S. lacustris estudiada durante dos ciclos anuales en Argentina, dónde se registró su pico de abundancia a fines del invierno y 


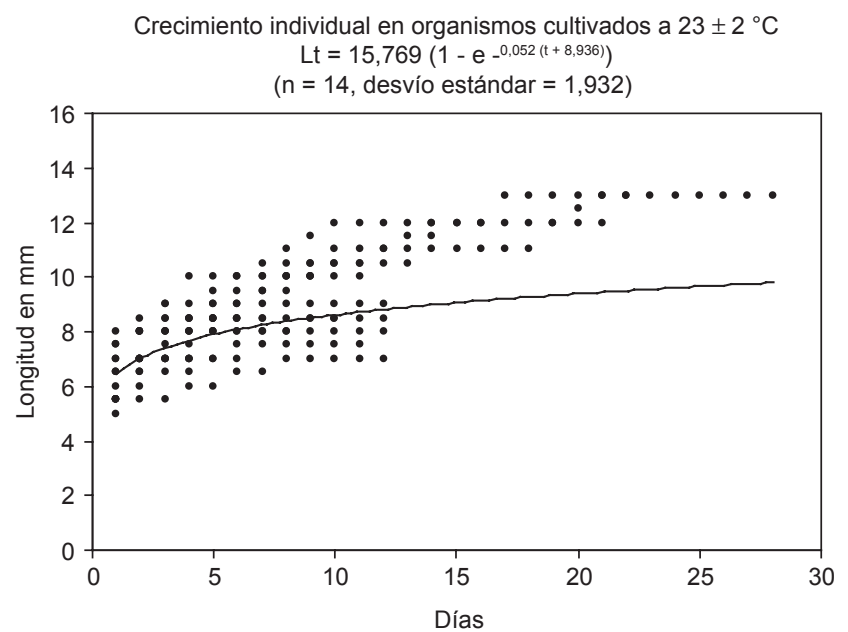

Crecimiento individual en organismos cultivados a $14 \pm 1{ }^{\circ} \mathrm{C}$ $\mathrm{Lt}=8,525\left(1-\mathrm{e}^{-0,057(\mathrm{t}+19,327)}\right)$ ( $n=16$, desvío estándar $=0,993$ )

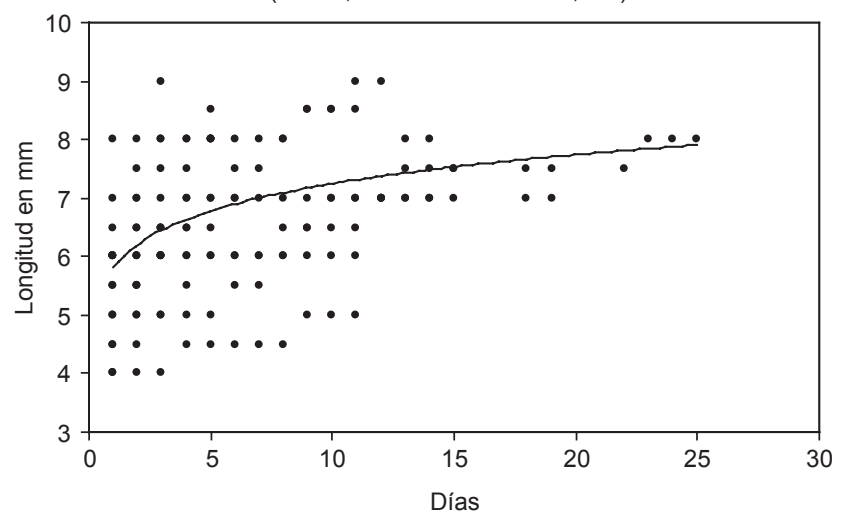

Figura 2. Crecimiento individual según la ecuación de von Bertalanffy en cultivos de Stylaria lacustris.

Figure 2. Individual growth according to von Bertalanffy equation in cultures of Stylaria lacustris.

principios de primavera (temperatura del medio entre $13-15{ }^{\circ} \mathrm{C}$ ) y su ausencia durante el verano, presentando de esta manera una clara estacionalidad (Armendáriz 2000). Las diferencias halladas entre las dos experiencias aquí realizadas concuerdan con ello, en cuanto el mayor número de zooides producidos por individuo se registró en los cultivos realizados a menor temperatura.

La longevidad media de $S$. lacustris en condiciones experimentales se estimó en 15-16 días, no hallándose diferencias significativas en los cultivos mantenidos a $14 \pm 1{ }^{\circ} \mathrm{C}$ de los mantenidos a $23 \pm 2{ }^{\circ} \mathrm{C}$. La supervivencia fue decreciendo gradualmente (según una curva de tipo 11l), mostrando de este modo una mortalidad proporcional. La expectativa de vida presentó su pico a los 16 días - edad en que se concentró una alta mortalidad- y luego se mantuvo aproximadamente constante hasta una edad de 28 días, a partir de la cual fue decreciendo en forma persistente hasta la última edad alcanzada.

En los cultivos los individuos se reprodujeron en forma asexual, formando cadenas de dos zooides, rara vez tres, como también registró Finogenova (1984), si bien indica un tiempo promedio empleado en la división de 4,2 días, algo menor al aquí registrado. Según Timm
(1984) las condiciones óptimas y estables de laboratorio hacen que los individuos no maduren sexualmente sino que se reproduzcan en forma asexual (aunque cada vez menos) llegando los clones a durar varios años en los cultivos. Finogenova (1984) no halló en sus cultivos individuos de $S$. lacustris que maduraran sexualmente. Sin embargo, durante esta investigación se observó la madurez sexual de los individuos mantenidos a $23 \pm 2{ }^{\circ} \mathrm{C}$, la cual pudo estar provocada por las condiciones de temperatura. Cabe señalar que en la población de S. lacustris estudiada en campo, los individuos maduros fueron hallados luego del pico de abundancia, cuando la temperatura del agua superó los $15^{\circ} \mathrm{C}$. Se estima, entonces que un aumento en temperatura podría actuar como un factor de estrés regulando la tasa de crecimiento e induciendo la madurez sexual de los individuos, los cuales fueron registrados en los cultivos mantenidos a mayor temperatura pero no en aquéllos mantenidos a $14 \pm 1^{\circ} \mathrm{C}$.

Los individuos que maduraron sexualmente presentaron una mayor longevidad que los inmaduros, viviendo en promedio casi el doble que estos últimos. Otros autores (Poddubnaya 1972, Timm 1984) registraron para esta especie un tiempo de vida de un mes para individuos inmaduros y 1-2 meses para los maduros. Luego de alcanzada la madurez sexual se produjo la muerte de los individuos. Distintos autores (Schierwater \& Hauenschild 1990a, b, Smith 1986) consideran que una vez alcanzada la madurez sexual, los individuos se reproducen una única vez y luego mueren. Si bien fueron hallados organismos clitelados que presentaban una zona de fisión, los mismos murieron antes de concluir la división asexual. La madurez sexual detendría de esta forma la reproducción asexual, como fuera observado por otros autores (Poddubnaya 1972, Timm 1984, Schierwater \& Hauenschild 1990a).

Los individuos cultivados a menor temperatura insumieron más tiempo en la reproducción asexual pero la longitud de la cadena de zooides al momento de la división fue mayor, similar a la registrada por Finogenova (1984). En cuanto a su crecimiento individual, los organismos mantenidos a mayor temperatura alcanzaron tallas mayores pero su tasa de crecimiento fue menor que la de aquellos mantenidos a menor temperatura.

\section{Referencias Bibliográficas}

ARMENDÁRIZ, L. C. 1999. Dinámica poblacional de Allonais lairdi (Oligochaeta, Naididae) en Los Talas, Provincia de Buenos Aires. Ecol. Austral 9:20-27.

ARMENDÁRIZ, L. C. 2000. Population dynamics of Stylaria lacustris (Linnaeus, 1767) (Oligochaeta, Naididae) in Los Talas, Argentina. Hydrobiologia 438:217-226.

BECKETT, D. C., AARTILA T. P. \& MILLER, A. C. 1992. Seasonal change in plant-dwelling Chironomidae and Naididae in a Wisconsin lake. J. Freshwat. Ecol. 7(1):45-57.

BRINKHURST, R. O. \& JAMIESON, B. G. M. 1971. Aquatic Oligochaeta of the World. Oliver y Boyd, Edinburgh.

BRINKHURST, R. O. \& MARCHESE, M. R. 1992. Guía para la identificación de oligoquetos acuáticos continentales de Sud y Centroamérica. Colección Climax $\mathrm{N}^{\circ}$ 6. Segunda edición, Argentina.

CHRISTENSEN, B. 1984. Asexual propagation and reproductive strategies in aquatic Oligochaeta. Hydrobiologia 115:91-95.

FINOGENOVA, N. P. 1984. Growth of Stylaria lacustris (L.) (Oligochaeta, Naididae). Hydrobiologia 115:105-107.

IRMLER, U. 1989. Population ecology and migration of Dero multibranchiata Stieren, 1892 (Naididae, Oligochaeta) in the Central Amazon inundation forest. Amazoniana 11(1):31-52.

JUGET, J. \& LAFONT, M. 1994. Theoretical habitat templets, species traits, and species richness: aquatic oligochaetes in the Upper Rhône River and its floodplain. Freshwat. Biol.31:327-340. 
LANG, C. 1990. Quantitative relationships between oligochaete communities and phosphorus concentrations in lakes. Freshwat. Biol. 24:327-334.

LEARNER, M. A., LOCHHEAD, G. \& HUGHES, B. D. 1978. A review of the biology of British Naididae (Oligochaeta) with emphasis on the lotic environment. Freshwat. Biol. 8:357-375.

LÉVÊQUE, C. 1971. Équation de von Bertalanffy et croissance des mollusques benthiques du Lac Tchad. Cah. O. R. S. T. O. M., ser. Hydrobiol., 5(3/4):263-283.

LIETZ, D. M. 1987. Potential for aquatic oligochaetes as live food in commercial aquaculture. Hydrobiologia 155:309-310.

LOCHHEAD, G \& LEARNER, M. A. 1983. The effect of temperature on asexual population growth of three species of Naididae (Oligochaeta). Hydrobiologia 98:107-112.

LODEN, M. 1981. Reproductive ecology of Naididae (Oligochaeta). Hydrobiologia 83(1):115-123.

MILBRINK, G. 1994. Oligochaetes and water pollution in two deep Norwegian lakes. Hydrobiologia 278:213-222.

PODDUBNAYA, T. L. 1972. [Particularidades de los ciclos vitales de Oligochaeta:Tubificidae y Naididae. Oligochaeta acuáticos (sistemática, ecología, investigaciones de la fauna de URSS). Acad. Cienc. URSS, Asoc. Hidrobiol. Sov. Trab.17. “Ciencia”, Moscú] [traducción del ruso].

SANG, Q. 1987. Some ecological aspects of aquatic oligochaetes in the Lower Pearl River (People's Republic of China). Hydrobiologia 155:199-208.
SÄRKKÄ, J. 1987. The occurrence of oligochaetes in the lake chains receiving pulp mill waste and their relation to eutrophication on the trophic scale. Hydrobiologia 155:259-266.

SCHIERWATER, B. \& HAUENSCHILD, C. 1990a. A photoperiod determined life-cycle in an oligochaete worm. Biol. Bull. 178:111-117.

SCHIERWATER, B. \& HAUENSCHILD, C. 1990b. The position and consequences of a vegetative mode of reproduction in the life cycle of a hydromedusa and an oligochaete worm. In: Advances in Invertebrate Reproduction 5 (Hoshi, M. \& Yamashita O. ,eds.). Elsevier Sci. Publ. B. V., Biomedical Division, p.37-42.

SLOREID, S. E. 1994. Oligochaete response to changes in water flow in the Dokka Delta, Lake Randsfjorden (Norway) caused by hydroelectric power development. Hydrobiologia 278:243-249.

SMITH, M. E. 1986. Ecology of Naididae (Oligochaeta) from an alkaline bog stream: life history patterns and community structure. Hydrobiologia 133:79-90.

SNEDECOR, G. W. \& COCHRAN, W. G. 1984. Métodos Estadísticos. C.E.C.S.A., México.

STREIT, B. 1978. A note on the nutrition of Stylaria lacustris (Oligochaeta: Naididae). Hydrobiologia 61:273-276.

TIMM, T. 1984. Potential age of Aquatic Oligochaeta. Hydrobiologia 115:101-104. 